\title{
DESIGN AND IMPLEMENTATION OF LOW COST DATA ACQUISITION SYSTEM FOR ECG SIGNAL AND EDR USING LABVIEW AND ARDUINO
}

\author{
Dilshad Hassan SALlo \\ Dept. Computer Science, College of Science, University of Duhok, Kurdistan Region-Iraq
}

(Received: January 29, 2019; Accepted for Publication: May 21, 2019)

\begin{abstract}
The usage of electrocardiogram (ECG) signals not only limited to the medical services, but also used for studying, learning, processing and analysing. However, the devices such as BIOPAC Systems that used for this purpose are extremely expensive and often not existed, particularly in the third world. The aim of this paper is to design and implement low cost data acquisition system to capture ECG signals and generating ECG-Derived Respiration (EDR) using the LabVIEW platform and Arduino with help of AD8232 chip. The system is designed to monitor the heart signals over the time and calculates the heart rate accurately. It also constructs the breathing signal indirectly by detecting R peaks in ECG signals and interpolating them. Furthermore, this system introduces a unique feature through giving ability to save both the heart and breathing signals as the Matlab file to be used for further analysis. The outcomes show that the ECG signals are in the standard range, as well as constructing breathing signal was successfully done, and the obtained low cost system can be useful for biomedical studies.
\end{abstract}

KEYWORDS: Arduino, DAQ, ECG signals, EDR, LabVIEW

\section{INTRODUCTION}

$\mathbf{E}^{c}$ $\mathrm{CG}$ is the process of recording the electrical activity of cardiac muscle over a period of time by putting electrodes over the skin (Puurtinen, Viik \& Hyttinen, 2008). This recording is implemented by Data acquisition system (DAQ) such as a Biopac system (Pflanzer and McMullen, 2012) which functions for capturing exhaustive data using sensors and displaying the findings through a graphical user interface (GUI). Data that is being recorded significantly used for detecting the heart problems and abnormal behavior. It also offers biomedical students' opportunity to learn analysing and studying fundamental features related to the heart. Unfortunately, such system can be elusive and cost around $\$ 4,000$ according to (Pflanzer and McMullen, 2012), which seems very expensive for many organizations and institutes. The purpose of this paper is to develop low cost data acquisition system for ECG signals and EDR which basically costs around $\$ 50$. The acquired data of DAQ are utilized for studying, processing and analysing by biomedical students. The proposed DAQ system contains three basic blocks as shown in figure 1 .

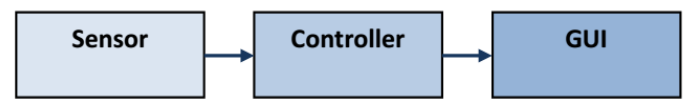

Fig. (1):- Basic Block Diagram of DAQ system

- Sensor used for an input data from human body by placing electrodes on the skin. These data are always in the form of voltage.

- Controller is worked as a communicator between real-world (sensor) and computer (GUI). After obtaining data using sensor and converted to analog output, data will be passed to the GUI by control to be displayed.

- GUI displays the acquired data as a graph on PC. 


\section{RELATED WORKS}

In the recent past, different approaches have been conducted for designing and implementing of data acquisition system for ECG signal. Some approaches used build-in DAQ hardware or other expensive chips to achieve this goal. In this section, the most relevant works to suggested approach are briefly presented with assigning their notable drawbacks. Authors (Bhat, Kumar \& Kumar, 2013) designed data acquisition system for ECG signal for multiple leads that able to get signal in three dimensions. This system has been used several costly chips to achieve its goal. It also limited only to ECG signal and neglecting Respiration signal. Authors (Wang, Niu \& Liu, 2013) presented ECG acquisition system based on Matlab, which allows acquiring signals and display them in Matlab GUI as well as calculating the heart rate mathematically. However, this system does not allow saving data for further study and analysis. It also not deals with respiration signal. Authors (Zhang, Sun \& Yang, 2012) provided significant ECG acquisition system, which able to transmit the ECG data using the wireless data transmission. However, this system does not provide a mechanism to save data as Matlab file, which is mostly used for analysing and processing. It also used elusive and expensive chips for accomplishing its goal. Authors (Kaur \& Sharma, 2014) presented system able to detect ECG components in LabVIEW and save analysed signals in the database. The major issue with this system is not able to acquire ECG signals in real time. It only focuses on analysing data that has been measured with other system. Authors (Gao, Wu, Zhou, Jiang \& Feng, 2012) designed data acquisition system for ECG signal with several features, namely high sampling rates and noise free, but it uses built-in DAQ to achieve its goal as well as this system cannot calculate and demonstrate heart rate accurately. Authors (BELGACEM, REGUIG, Naït-Ali \& Fournier, 2012) presented system able to detect not only $R$ peaks but also $\mathrm{P}$ and $\mathrm{T}$ peaks of ECG. It also used database for storing ECG signals and components in a simple manner. Major issue with this system is not dealing with breathing signal directly or indirectly as well as using an expensive DAQ to acquire the ECG signal.

After presenting several previous works that are related to the subject of the proposed research and showing backwards features that are not covered in previous works, this research provides significant functionalities that make capturing and displaying signals easy as well as introducing extraordinary features that not existed in any previous search namely allowing the system to construct a respiration signal without sensor.

\section{SYSTEM COMPONENTS}

\subsection{SENSOR}

AD8232 chip is a single lead heart used to measure the electrical activity of the heart by calculating the difference of voltages between a negative electrode and a positive electrode and obtained result of the voltages is converted to analog signal as an output to be transmitted to Arduino. AD8232 provides on board three pins namely RA (Right Arm), LA (Left Arm), and RL (Right Leg) correspond the electrodes that be attached on a subject's body for measuring the heart signals (Ji, Ning, Zhao \& Zhang, 2017) . It also provides essential pins such as SDN, LO+, LO-, OUTPUT, 3.3V, GND for operating this monitor with an Arduino or other development board.

\subsection{CONTROLLER}

Arduino Uno is used as a microcontroller in this system. This board is based on the ATmega328P. The board is equipped with sets of digital and analog input/output (I/O) pin. The board has 14 Digital pins, 6 analog pins as well as containing all needed to support the microcontroller; by connecting it to a computer with a USB cable or power it with an AC-to DC adapter or battery to get started (Kanani \& Thakker, 2015). Arduino receives signal from the sensor; after applying a low pass filter with cutoff frequency of $1 \mathrm{~Hz}$ to remove artifacts from movements, then obtained signal is transmitted to LabVIEW, which runs on the PC to be displayed properly.

\subsection{GUI}

On PC, GUI is run using LabVIEW 2015, which is a development environment that used for virtual programming language (Kim, 2011). The graphical user interface of system is designed using LabVIEW for displaying ECG signals and EDR signal as well as giving control over the system. It also provides a block diagram coding that works beyond the interface to tell the system what to do exactly. 


\section{BLOCK DIAGRAM AND DESCRIPTION}

The block diagram of system is shown in figure (2). The system has one sensor AD8232, which able to obtain data from single lead of the body of human in once session. Obtained data will transfer to Arduino as analog output. The data received on serial monitor of Arduino is serially transmitted to PC with help of serial communication cable. Then, data will be displayed on GUI that has built using LabVIEW to fit the purpose. The serial data acquisition is done using the Virtual Instrument Software Architecture (VISA) tool. VISA is a protocol to provide communication between LabVIEW and any other device. Here the data is received serially at 9600 baud rate. On execution of the code, the values will get obtain on the serial monitor window in Arduino software. The number of bytes of data will be depending on the string length. Now, this data will get transferred to LabVIEW by serial port. When data reached to it, they can be exploited to offer extraordinary feature namely reconstruct respiration signal using $R$ peaks in ECG signal. Due to respiration signal is one of the biosignals that can be measured indirectly (Klein, 2015). Because of breathing activity affect the position of the apex of the heart (Pflanzer and McMullen, 2012). In inspiration mode, the apex of the heart gets close to mean electrical axis, which leads to raise the peaks of R. In contrast, expiration mode forces the apex of the heart to go far from mean electrical axis, leading to decline the peaks of R in ECG signal (Dickhaus \& Maier, 2010).

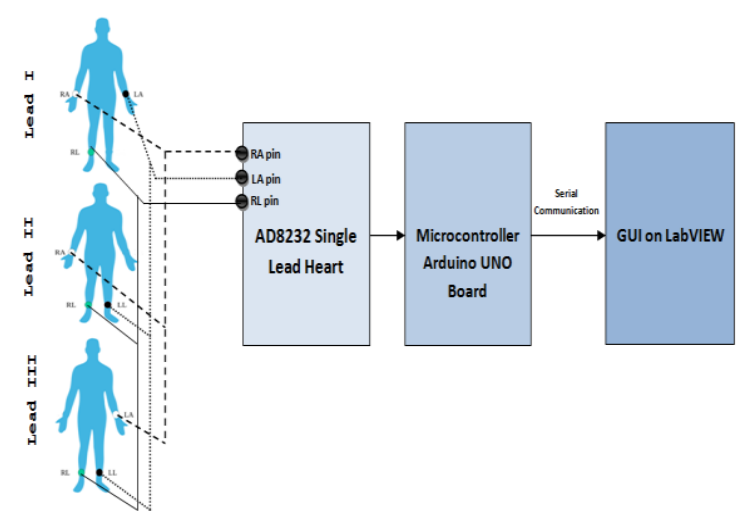

Fig. (2):- Block Diagram of our System

As a result, the whole ECG signals are oscillated according to inspiration and expiration phases as shown in figure (3) (Moody, George, Mark, Roger, Zoccola \& Mantero, 1985). This influence over $\mathrm{R}$ peaks representing respiration cycles and can be extracted with help of programming.

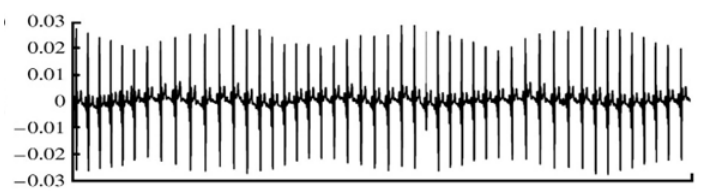

Fig. (3):- ECG signals affected by breathing activity

\section{EXPERIMENTAL SETUP AND FLOWCHART OF ACQUISITION PROCESS}

Measuring the ECG signals requires a negative, a positive and a ground electrodes which already been set up as an input to (LO+, LO-, GND) in the sensor respectively. The acquisition process commences by determining which single lead will be chosen, then the system will calculate the difference of voltage to be transmitted as signal through input to pin A0 in Arduino as shown in figure(4). The microcontroller receives signals and has control over them. When the processing of signals is done, they will be sent to PC using serial communication to be displayed by the graph of LabVIEW. In fact, the Arduino considered as a bridge between the real world 
represented by sensor and interface represented by LabVIEW. When the signal reached to LabVIEW, the system detects all $\mathrm{R}$ peaks with help of programming, and resampling them based on original sample rate to generate breathing signal and demonstrate on graph as well. As a result, the breathing signal is extracted and displayed in GUI without expensive redundant sensor.

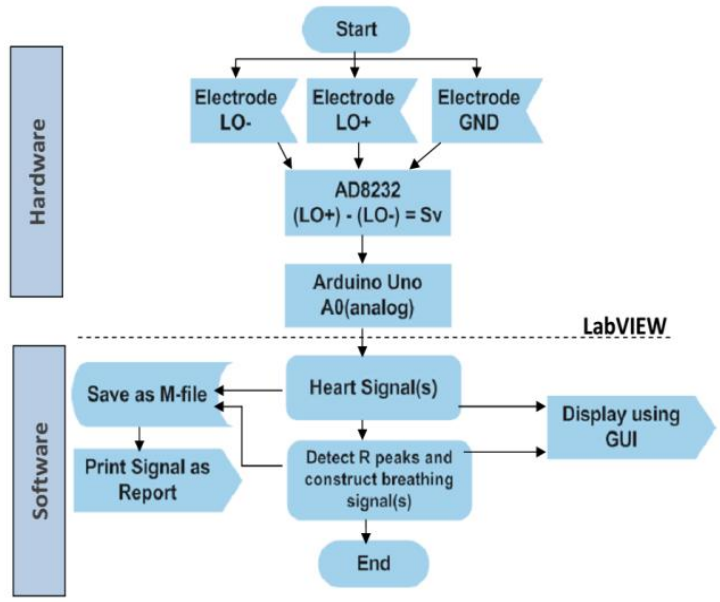

Fig. (4):- Flowchart of Acquisition System

The actual setup of the system is shown in figure (5), which demonstrates all equipment used.

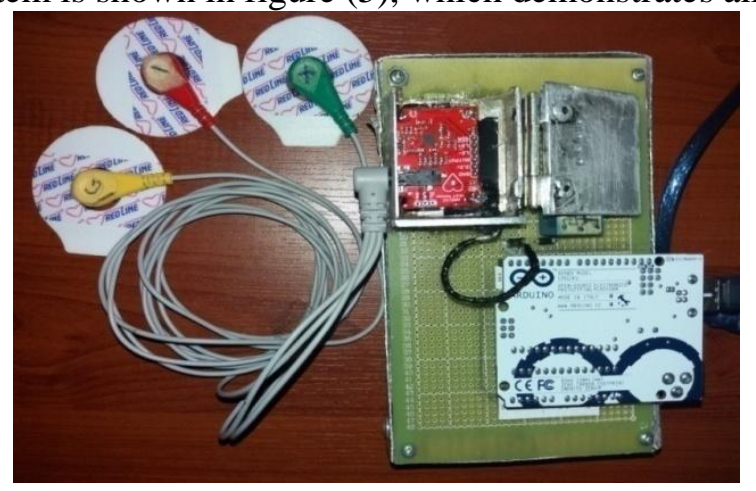

Fig. (5):- Experimental Setup and Equipment of System

Here in figure (6), LabVIEW GUI is shown. It displays the obtained signals on PC and it also has control over the sensor.

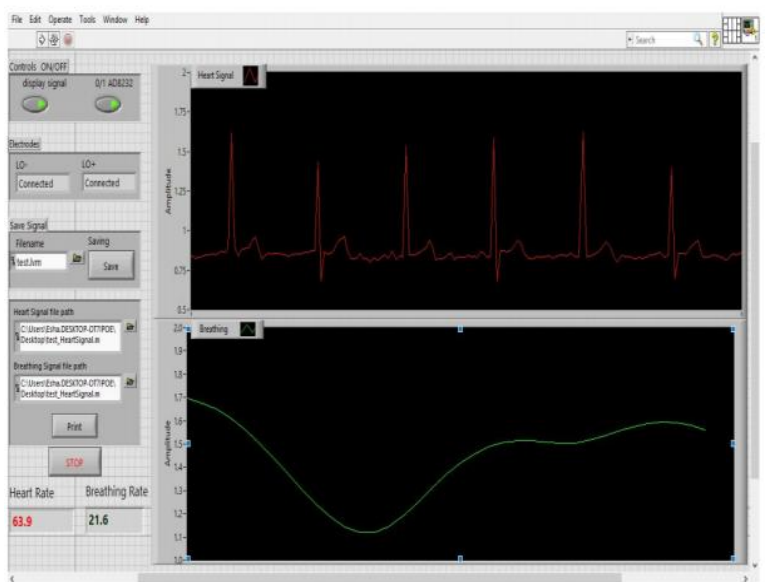

Fig. (6):- LabVIEW Front Panel GUI 
Furthermore, it gives privilege to a user to save both signals ECG and breathing as Matlab files for studying, analysing as well as ability to print them.

\section{ANALYSIS AND RESULT}

The low cost data acquisition system for ECG signal and EDR was tested with Lead I, Lead II and Lead III, on 32 Subjects, 6 Subjects (18.75\%) were female and the remaining 26 Subjects $(81.25$ $\%$ ) were male, their ages ranged from 19 to 23 years. When the signals of both ECG and EDR had been measured and saved as Matlab-files, the analysing phase has been conducted using Matlab 2017 on obtained signals. The accuracy of the system was verified through the results of Subjects were shown within the standard heart rate $75 \mathrm{BPM}$ as shown in table 1 . The system shows good result within standard range and can be useful to obtain data for studying and other purposes.

Table (1): Result of ECG components for 32 Subjects.

\begin{tabular}{|c|c|c|c|}
\hline \multicolumn{4}{|c|}{$\begin{array}{c}\text { Normative Value Based on Heart Rate } 75 \\
\text { BPM }\end{array}$} \\
\hline \multicolumn{4}{|c|}{$\begin{array}{l}\text { ECG Component } \\
\text { Duration }(\mathrm{sec})\end{array}$} \\
\hline & $\mathbf{P}$ & QRS & $\mathbf{T}$ \\
\hline Subje & $.07-$ & $.06-$ & .10 \\
\hline cts & .18 & .12 & .25 \\
\hline 1 & 0.079 & 0.07 & 0.133 \\
\hline 2 & 0.081 & 0.063 & 0.129 \\
\hline 3 & 0.066 & 0.073 & 0.134 \\
\hline 4 & 0.08 & 0.094 & 0.124 \\
\hline 5 & 0.083 & 0.087 & 0.135 \\
\hline 6 & 0.076 & 0.103 & 0.111 \\
\hline 7 & 0.136 & 0.087 & 0.182 \\
\hline 8 & 0.124 & 0.091 & 0.185 \\
\hline 9 & 0.084 & 0.072 & 0.122 \\
\hline 10 & 0.07 & 0.071 & 0.111 \\
\hline 11 & 0.069 & 0.065 & 0.107 \\
\hline 12 & 0.072 & 0.067 & 0.124 \\
\hline 13 & 0.076 & 0.079 & 0.141 \\
\hline 14 & 0.115 & 0.091 & 0.202 \\
\hline 15 & 0.073 & 0.09 & 0.098 \\
\hline 16 & 0.077 & 0.086 & 0.094 \\
\hline 17 & 0.122 & 0.097 & 0.173 \\
\hline 18 & 0.073 & 0.089 & 0.104 \\
\hline 19 & 0.092 & 0.081 & 0.151 \\
\hline 20 & 0.063 & 0.095 & 0.12 \\
\hline 21 & 0.082 & 0.086 & 0.115 \\
\hline 22 & 0.071 & 0.084 & 0.106 \\
\hline 23 & 0.069 & 0.096 & 0.098 \\
\hline 24 & 0.102 & 0.095 & 0.192 \\
\hline 25 & 0.09 & 0.077 & 0.134 \\
\hline 26 & 0.086 & 0.072 & 0.127 \\
\hline 27 & 0.071 & 0.076 & 0.127 \\
\hline 28 & 0.087 & 0.072 & 0.149 \\
\hline 29 & 0.079 & 0.077 & 0.127 \\
\hline 30 & 0.095 & 0.074 & 0.166 \\
\hline 31 & 0.084 & 0.082 & 0.167 \\
\hline 32 & 0.077 & 0.068 & 0.15 \\
\hline
\end{tabular}




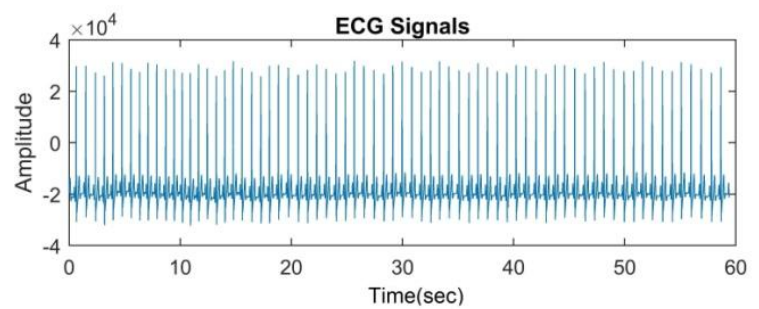

Fig. (9):- Signals for One of the Subjects Displaying Using Matlab

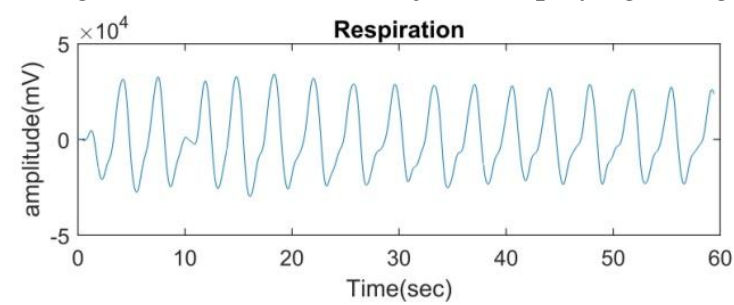

Fig.(10):- Breathing Signals generated by Subject's ECG Signals

As breathing sensor is not used in our system, $\mathrm{R}$ peaks method which had been approved according to (Sarkar, Bhattacherjee \& Pal, 2015). The verification of breathing has done using counting; the system uses equation below to calculate breathing rate by detecting highest peaks and finds delta time (Ruangsuwana, Velikic \& Bocko, 2010).

$\mathrm{BR}=(60 / \Delta$ PeakTime $)[$ breaths $/$ minute $] ..$. (1)

The same equation is also used to find heart rate, but using $\mathrm{R}-\mathrm{R}$ interval with delta time.

$\mathrm{HR}=(60 / \Delta$ PeakTime $)[$ beats/minute $]$

\begin{tabular}{|l|c|}
\hline Heart Rate & Breathing Rate \\
\hline 63.9 & $\mathbf{2 1 . 6}$ \\
\hline
\end{tabular}

Fig.(11):- Displaying Heart Rate and Breathing Rate.

Best result of breathing signals can be obtained by training the subject to do inspiration and expiration phases slowly as well as choosing Lead II because it has prominent $\mathrm{R}$ peaks which affected significantly by breathing as well as easily detected for generating breathing signals.

\section{CONCLUSION AND FUTURE WORK}

In the above work, we had proposed low cost DAQ system using Arduino-UNO controller and LabVIEW GUI. The system is able to measure heart electrical signals efficiently as well as extracting breathing signals without extra sensor. It also finds heart rate and breathing rate by calculating delta time between two successive peaks. The proposed system can be useful for biomedical study as well as offering opportunity to acquire real ECG signals that are enriching information about the heart. Further work can be added and implemented to the system for enhancing its functionalities such as adding two more sensors to measure not only one lead of ECG signals; instead, three leads of ECG signals, and getting complete information in three dimensions of the heart.

\section{REFERENCES}

-BELGACEM, N., REGUIG, F., Naït-ali, A., \& Fournier, R. (2012). Person Identification System Based on Electrocardiogram Signal Using LabVIEW. International Journal on Computer Science and Engineering (IJCSE), 4, $974-981$.

-Bhat, A., Kumar, V., \& Kumar, S. (2013). Design of ECG Data Acquisition System. International Journal of Advanced Research in Computer Science and Software Engineering, 3(4), 676680 .

- Dickhaus, H., \& Maier, C. (2010). Central Sleep Apnea Detection from ECG-derived Respiratory 
Signals. Methods Of Information In Medicine, 49(05), 462-466. doi: 10.3414/me0902-0047.

- Gao, Z., Wu, J., Zhou, J., Jiang, W., \& Feng, L. (2012). Design of ECG Signal Acquisition and Processing System. 2012 International Conference On Biomedical Engineering And Biotechnology. doi: 10.1109/icbeb.2012.128.

- Ji, X., Ning, C., Zhao, C., \& Zhang, X. (2017). Design of the HRV Analysis System Based on AD8232. Destech Transactions On Engineering And Technology Research, (ismii). doi: 10.12783/dtetr/ismii2017/16676.

- Kanani, N., \& Thakker, M. (2015). Low Cost Data Acquisition System Using LabVIEW. 2nd International conference on multidisciplinary research \& practice.

- Kaur, P., \& Sharma, R. (2014). LabVIEW based design of heart disease detection system. International Conference On Recent Advances And Innovations In Engineering (ICRAIE2014). doi: 10.1109/icraie.2014.6909241

- Kim, N. (2011). Digital Signal Processing SystemLevel Design Using LabVIEW. Newnes.

- Klein, R. (2015). Editorial: Derivation of respiratory gating signals from ECG signals. Journal of Nuclear Cardiology, 23(1), 84-86. doi: 10.1007/s12350-015-0192-6.

- Moody, George, B., Mark, Roger, G., Zoccola, A., \& Mantero, S. (1985). Derivation of Respiratory
Signals from Multilead ECGs. Computers in Cardiology, 12.

- Pflanzer, R. and McMullen, W. (2012). Electrocardiography (ECG) I Introduction. Biopac Student Lab Manual. BIOPAC Systems, Inc., pp.pp. I1-I4.

- Puurtinen, M., Viik, J., \& Hyttinen, J. (2008). Best Electrode Locations for a Small Bipolar ECG Device: Signal Strength Analysis of Clinical Data. Annals Of Biomedical Engineering, 37(2), 331-336. doi: 10.1007/s10439-008-9604-y.

- Ruangsuwana, R., Velikic, G., \& Bocko, M. (2010). Methods to extract respiration information from ECG signals. In Conf. Proc. ICASSP (pp 5703).

- Sarkar, S., Bhattacherjee, S., \& Pal, S. (2015). Extraction of respiration signal from ECG for respiratory rate estimation. Michael Faraday IET International Summit 2015. doi: 10.1049/cp.2015.1654.

- Wang, X., Niu, W., \& Liu, Y. (2013). Design of ECG Acquisition System Based on Matlab. Applied Mechanics And Materials, 462-463, 1001-1004. doi: $10.4028 /$ www.scientific. net/amm.462463.1001.

- Zhang, Y., Sun, G., \& Yang, Y. (2012). 12-Lead ECG Data Acquisition System Based on ADS1298. Procedia Engineering, 29, 21032108. doi: 10.1016/j.proeng.2012.01.270.

تم في هذه الدراسة تصميم وتنفيذ نظام الحصول على البيانات لـ اشارات القلب والتنفس بتكلفه قليلة جداً مقارنتا بـ الانظمة المصصمة لهذا الغرض. هذا النطام استخدم Arduino and LabVIEW مع مساعدة للحصول على البيانات من جسم الانسان. هذا النظام يقوم بمراقبة اشارات القلب على مدار

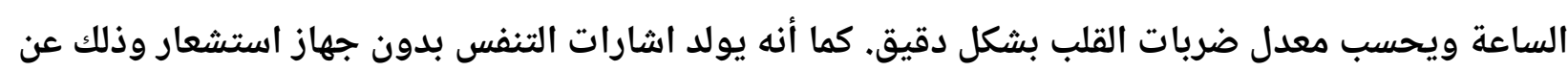

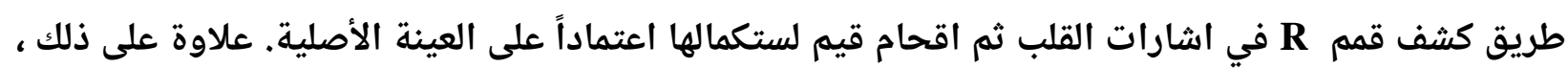

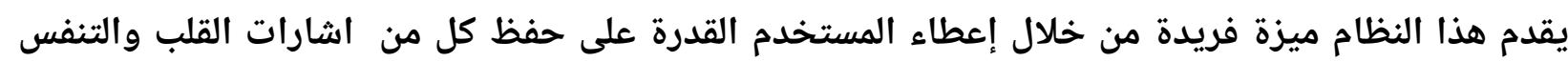
كملف Matlab لاستخدامها في التحليل والدراسة من قبل قسم الهندسة الطبية الحيوية. 